\title{
Fault Detection Considerations in Silicon Based MEMS Resonators by Observing Changes in Dynamic Behaviour
}

\author{
S. İkizoğlu and M. Akyol
}

\begin{abstract}
This study is about fault detection in siliconbased MEMS resonators. The main idea in finding out the failure is to establish a proper relationship between the mechanical structure and its electrical equivalent and prosecuting related measurements. In order to determine the type of defect, the electrical equivalent circuit is referenced considering the parasitic effects. Among various possible faults cracks in the beam and particle adhesion are selected to verify the validity of the approach. Simulations are carried out to study the effect of defects on the resonance frequency and amplitude. Results coincide greatly with those of similar investigations giving motivation for further studies to penetrate deep into the matter, thus not being restricted with defining the trouble, but even locate the failure.
\end{abstract}

Index Terms- MEMS Resonator, resonance frequency, quality factor, transduction factor

\section{INTRODUCTION}

$\mathrm{D}^{2}$ AY by day the application of MEMS devices is growing up rapidly in various areas due to their superior advantages such as low power consumption, small size, long life and low cost [1]. Among numerous application areas MEMS resonators find also remarkable place [2-4]. This is because the MEMS resonators offer excellent properties than their IC-counterparts with their long-term stability and extremely high quality factor [5]. Thus, they are widely used in oscillators and filters.

On the other side, with the increasing demand toward MEMS devices, the requirement for reliability is also ascending rapidly. Concerning this issue, several precautions are taken and measurements are carried out during the fabrication process. Additionally, observations are made in dynamic conditions. Investigations put forward that there are several reasons which cause malfunction of MEMS resonators. Among all, faults caused by particle adhesion and cracks are at the top of the list [6]. Besides these, friction due to particle contaminants, fatigue of the material, deformation, etching faults etc. also lead to failure. Several studies have been carried out to describe the relationship between the defect and the corresponding parameter [7-10].

S. İkizoğlu is with the Department of Control and Automation Engineering, Istanbul Technical University, Istanbul, Turkey (e-mail: ikizoglus@itu.edu.tr).

M. Akyol is with Turkish Airlines Technique Inc., Turkey.
In this paper we will mainly focus on faults caused by cracks and particle adhesion, but the way we define the source of the trouble is also valid to find out of others. Any defect in the structure leads to changes in the natural frequency of the MEMS resonator, in the amplitude of the vibration or in the value of the quality factor. Thus, representing the electrical circuit with its y-parameters will be helpful for the analysis. The parameter $y_{21}$ relates the output current to the input voltage giving the highest value at the resonance frequency.

\section{MEMS RESONATOR MODEL}

There are various structures for MEMS resonators. While the comb structure is becoming popular in recent years, micro-flap and bridge resonators are also used in different applications [11]. Since for all structures we speak of 'distributed' mass and elasticity, MEMS resonators are investigated using the 'lumped' mass-spring or electricalequivalent model. The mass-spring model (Fig. 1a) has its well- known equation as

$m \ddot{x}+\gamma \dot{x}+k x=F$

where $\mathrm{m}$ represents the effective mass and $\gamma$ and $\mathrm{k}$ stand for damping constant and effective spring constant, respectively; $\mathrm{F}$ is the external force acting on the system. Here,

$k=\omega_{0}^{2} m$

where $\omega_{0}$ is the first natural frequency of the resonator beam. The mass-spring model can be related to the electrical model (Fig.1b) with following equivalencies:

$\dot{x}=i, \gamma=R, m=L, k=1 / C, F=u$

where $u$ is the voltage applied, and $i$ is the current through the series circuit.

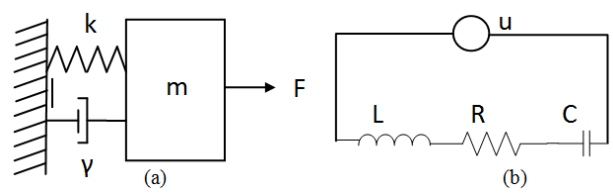

Fig.1. a) Mass-spring model b) Electrical equivalent 
In MEMS resonators, first the excitation voltage is converted into mechanical vibration which is further converted into current $\mathrm{i}$ defined in the electrical equivalent circuit. This capacitive transduction can be formulated as follows:

$\mathrm{U}=\mathrm{U}_{\mathrm{DC}}+\mathrm{U}_{\mathrm{AC}}$ is applied to the resonator, where $\mathrm{U}_{\mathrm{DC}}$ serves for two the purposes: The one is that the resonator does not oscillate at twice the fundamental resonance frequency [12]. It further is directly effective on the transduction. The capacitance $\mathrm{C}_{0}$ of the capacitor that is formed by the base electrode and the beam changes due to the excitation voltage $\mathrm{U}$ as

$C=\varepsilon \frac{A}{d-x}=C_{0} \frac{d}{d-x}$

with $\mathrm{C}_{0}=\varepsilon \mathrm{A} / \mathrm{d}$, where $\mathrm{A}$ is the effective area, $\varepsilon$ the dielectric constant, $d$ the plate separation and $x$ the change in $d$ due to exerted electrostatic force. Thus, the energy of the capacitor is $W=C U^{2} / 2$ and the force acting onto the beam is given as

$F=\frac{\partial W}{\partial x}=\frac{1}{2} \frac{\varepsilon A}{(d-x)^{2}} U^{2}$

Assuming $\mathrm{x}<<\mathrm{d}$, the AC component of this force can be approximately calculated as

$F_{A C}=\frac{U_{D C} U_{A C} C_{0}}{d}=\eta U_{A C}$

Here,

$\eta=U_{D C} C_{0} / d$

is called the transduction factor. Since the current is defined as $\mathrm{i}=\partial \mathrm{Q} / \partial \mathrm{t}(\mathrm{Q}$ : charge) and for a capacitor $\mathrm{C}=\partial \mathrm{Q} / \partial \mathrm{U}$, the current through the resonator can be expressed as

$i=\frac{\partial(C U)}{\partial t}=U \frac{\partial C}{\partial t}+C \frac{\partial U}{\partial t} \approx \eta \frac{\partial x}{\partial t}+C_{0} \frac{\partial U_{A C}}{\partial t}$

Consequently the electrical equivalent circuit of the resonator can be drawn as in Fig.2.

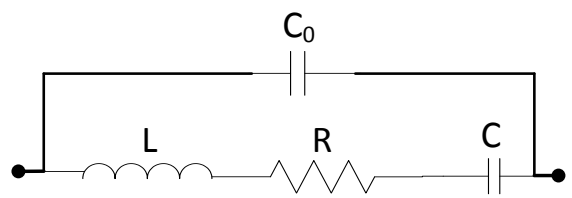

Fig.2. Electrical equivalent circuit of a MEMS resonator

In Fig.2 following equivalencies are valid [13]:

$L=\frac{m_{e f f}}{\eta^{2}}, C=\frac{\eta^{2}}{k_{e f f}}, R=\frac{\sqrt{k_{e f f} m_{e f f}}}{Q \eta^{2}}$
Here $\mathrm{m}_{\mathrm{eff}}$ and $\mathrm{k}_{\mathrm{eff}}$ stand for effective mass and effective stiffness of the resonator, respectively; $Q \approx m \omega_{0} / \gamma$ represents the quality factor. At this point it is worth to note that the effective spring constant has two main components and is given as

$k_{e f f}=k_{i}-k_{s}$

where $\mathrm{k}_{\mathrm{i}}$ is the initial spring constant and $\mathrm{k}_{\mathrm{s}}$ represents the softening effect given by

$k_{S} \approx \frac{\varepsilon_{0} A_{e f f} U_{D C}^{2}}{d^{3}}$

with $A_{\text {eff }}$ being the exposed area of the beam and $d$ the plate separation [13].

\section{MODEL INCLUDING PARASITIC EFFECTS}

\section{A. Resonator Model}

The common used model of a MEMS resonator is as in Fig.2. Fig. 3 shows the cross section of a micro-flap resonator [14]. Here, $\mathrm{C}_{\mathrm{p} 1}$ and $\mathrm{C}_{\mathrm{p} 2}$ represent shunt capacitances of field oxide between the electrodes and the substrate. Thus, Tanaka et al suggest that according to Fig. 3 the electrical equivalent circuit of a resonator should be constructed as in Fig. 4 which takes the parasitic effects into consideration [14]. Here, $C_{\text {pad }}$ represents the shunt capacitance between $\mathrm{Si}$-substrate and the measurement pad and $\frac{1}{C_{p}}=\frac{1}{C_{p 1}}+\frac{1}{C_{p 2}}$.

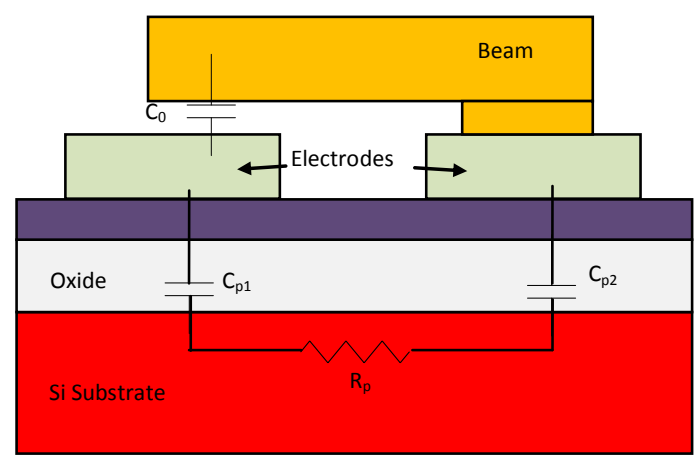

Fig.3. Cross section of a micro-flap resonator.

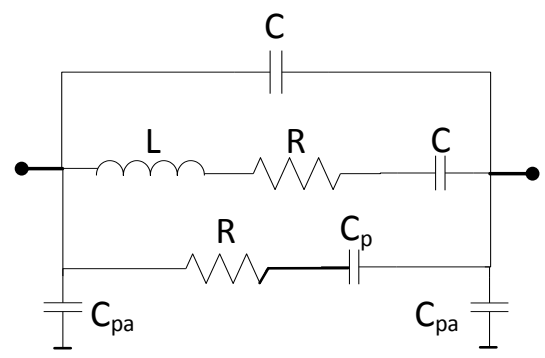

Fig.4. Equivalent circuit of the MEMS resonator considering parasitic effects. 


\section{B. y-parameter Equivalent Circuit}

For the equivalent circuit in Fig. 4 it is obvious that at the resonance frequency of the resonator, $\mathrm{y}_{12}\left(=\mathrm{y}_{21}\right)$ parameter will have its highest value. For this circuit the expression for $\mathrm{y}_{12}$ can be derived as follows:

$y_{12}=\frac{A s^{4}+B s^{3}+D s^{2}+E s}{F s^{3}+G s^{2}+H s+1}$

where

$$
\begin{aligned}
& A=R_{p} C_{0} C_{p} L C \\
& B=R_{p} C_{0} C_{p} R C+C_{0} L C+C_{p} L C \\
& D=C_{p} R_{s}+C_{0} R_{p} C_{p}+C_{0} R C+C C_{p} R_{p} \\
& E=C_{0}+C+C_{p} \\
& F=R_{p} C_{p} L C \\
& G=R_{p} R_{s} C_{p} C+C L \\
& H=C_{p} R_{p}+R C
\end{aligned}
$$

\section{EFFECT OF FAULTS ON CHARACTERISTIC CURVES}

In order to examine the effect of the changes in several parameter values on the resonance frequency, a set of initial values are taken as reference with:

$\mathrm{C}_{0}=25 \mathrm{e}-15 \mathrm{~F} ;$

$\mathrm{C}_{\mathrm{p}}=20 \mathrm{e}-15 \mathrm{~F}$

$\mathrm{C}_{\mathrm{pad}}=0.8 \mathrm{e}-12 \mathrm{~F}$

$\mathrm{C}=1 \mathrm{e}-15 \mathrm{~F}$

$\mathrm{R}=100 \mathrm{e} 3 \Omega$;

$\mathrm{L}=100 \mathrm{e}-3 \mathrm{H}$

$\mathrm{R}_{\mathrm{p}}=0.01 \Omega$.

These values give a resonance frequency of $\mathrm{f}_{0}=15.883 \mathrm{MHz}$, while this frequency would be $15.915 \mathrm{MHz}$ for the equivalent circuit in Fig.2.

Below is investigated how several deviations from the initial values would affect $\mathrm{f}_{0}$.

Change in transductance $\eta$ : The relative sensitivity of $\mathrm{L}$ toward $\eta$ is $\partial \mathrm{L} / \partial \eta / \mathrm{L}=-2 / \eta$. Thus, a change of $1 \%$ in $\eta$ results in a change of $-2 \%$ in $\mathrm{L}$. On the other side, a change in $\eta(\Delta \eta)$ leads to a change in $\mathrm{C}$ as $\Delta \mathrm{C} / \mathrm{C}=2 \Delta \eta / \eta$, thus cancelling the effect of the change in $\mathrm{L}$ for the resonance frequency, if the equivalent circuit is taken as in Fig.2. But this will not be case, if the change in $\eta$ is due to a change in the polarization voltage $\mathrm{U}_{\mathrm{DC}}$, because $\mathrm{U}_{\mathrm{DC}}$ is also effective in $\mathrm{k}_{\mathrm{s}}$. Considering the equivalent circuit as in Fig.2, the resonance frequency is calculated as,

$$
\omega_{0}=\frac{1}{\sqrt{L C}}=\sqrt{\frac{k_{e f f}}{m_{e f f}}}=\sqrt{\frac{k_{i}}{m_{e f f}}\left(1-\frac{k_{s}}{k_{i}}\right)}
$$

As $\mathrm{R}$ is also dependent on $\mathrm{k}_{\text {eff }}$, not only the resonance frequency, but also the amplitude of the curve will be affected. Fig.5 shows the dependency of the resonance frequency on $\mathrm{U}_{\mathrm{DC}}$ with the assumption that for the initial case $\mathrm{U}_{\mathrm{DC}}=10 \mathrm{~V}$.

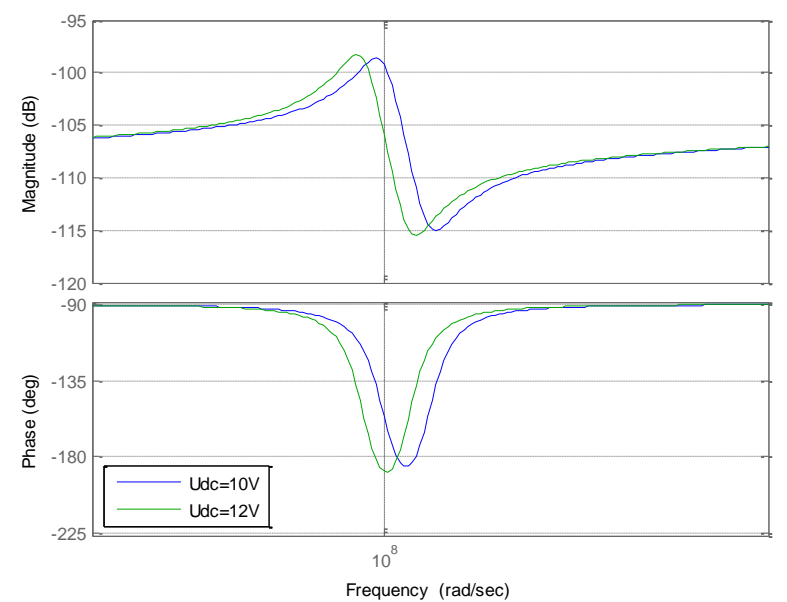

Fig.5. Effect of the polarization voltage upon the resonance frequency and amplitude

Change in effective stiffness: A crack in the beam will decrease its stiffness. If we assume that the effective mass is being kept constant, the decrease in stiffness increases $\mathrm{C}$ and reduces $\mathrm{R}$ according to Equations in (8). The result is demonstrated in Fig.6.

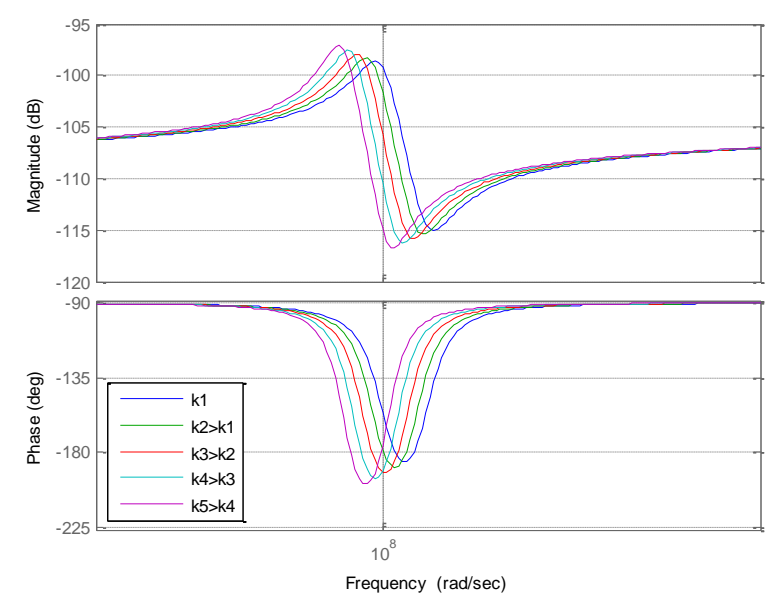

Fig.6. Effect of the effective stiffness $k$ upon the resonance frequency due to a beam crack.

If a particle adhesion fault exists, the effective stiffness will increase and accordingly an increase in frequency eventuates. This condition is illustrated in Fig.7. We clearly observe that besides the increase in frequency, the amplitude decreases, thus reducing the sensitivity.

A particle adhesion will also decrease $\mathrm{C}_{0}$ since it effects the separation $d$ between the base and the beam. The change of the frequency characteristic only due to $\mathrm{C}_{0}$ is illustrated in Fig.8. It is observed that besides a slight shift in the resonance frequency, mainly the overall amplitude decreases with decreasing $\mathrm{C}_{0}$. 


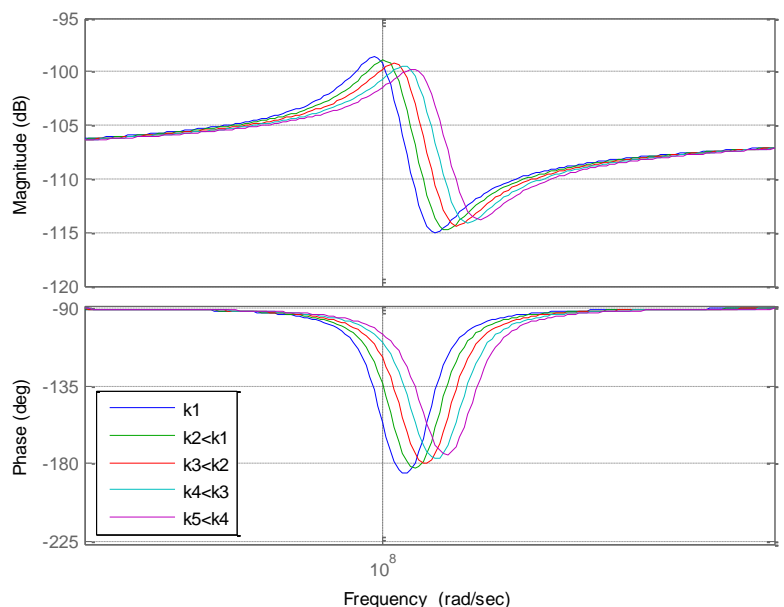

Fig.7. Effect of the effective stiffness $k$ upon the resonance frequency due to particle adhesion fault.

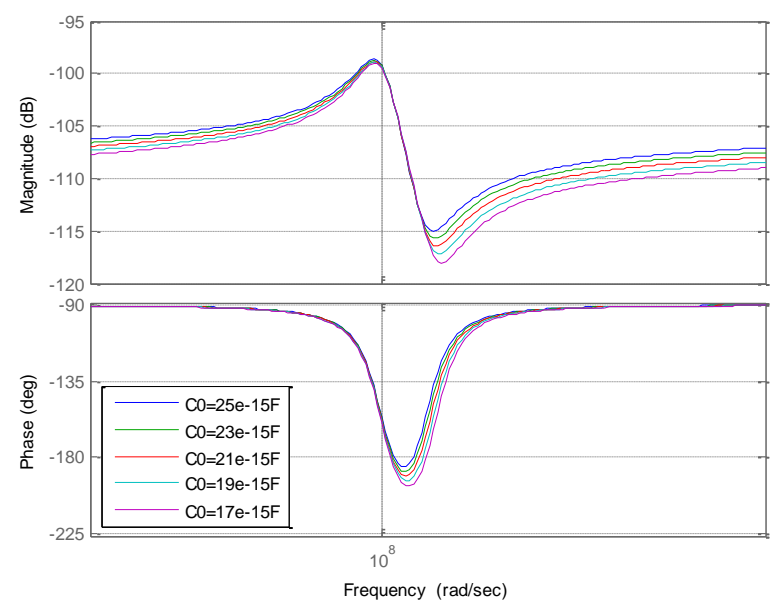

Fig. 8. Effect of $\mathrm{C}_{0}$ on the frequency characteristic of the resonator.

\section{CONCLUSION AND FUTURE WORK}

In this study fault detection approach for silicon based MEMS resonators is handled. For this purpose, an electrical equivalent circuit is related to the mechanical spring-mass model of the resonator, where parasitic effects are also taken into account. The main idea is based upon the fact that any defect in the structure leads to a certain shift of the resonance frequency and change in the amplitude and quality factor. Mainly the effect of two defects -crack in the beam and particle adhesion fault- is analyzed. The results coincide greatly with those of other studies $[6,15,16]$. This is considered as a proof of the validity of the approach and encourages for extending the studies to cover other possible faults. The next step will be to define more precisely the relationship between the structural troubles and related changes in the electrical equivalent circuit. It is clear that a fault may influence several parameters. On the other side different defects may be effective on the same parameter in different proportions. Hence, a detailed investigation with experimental approval is needed in order to concretize the fault.

This study has mainly fucused on the way how to approach to find out the reason of the malfunction of a MEMS resonator. On this way several assumptions have been made such that the nonlinearities of the spring constant in the mechanical equivalent structure have been disregarded. Future work will cover the deep study taking as many effective parameters into consideration as possible. A major part of future work should also be constituted by the interrelation between the location of the defect and the corresponding deviation in functioning of the resonator.

\section{REFERENCES}

[1] S. D. Senturia, "Microsystem Design", Kluwer Academic Publishers, $2^{\text {nd }}$ ed., Massachusetts, 2001.

[2] T. Clark, C. Nguyen, L. P. B. Katehı, G. M. Rebeı, "Micromachined devices for wireless communications", Proc. of the IEEE, vol. 86, no. 8, pp 1756-1768, Aug. 1998.

[3] W.T. Hsui, T. Clark, C. Nguyen, "Stıffness-compensated temperature-insensitive micromechanical resonators", Proc. of the IEEE, pp. 731-734, 2002.

[4] N. Sepulveda, D. Aslam, J.P. Sullivan, "Polycrystalline diamond MEMS resonator technology for sensor applications", Diamond \& Related Materials, Elsevier, vol. 15, pp 398 - 403, 2006.

[5] V. Kaajakari, "Theory and analysis of MEMS resonators", VTI Technologies, 2011.

[6] A. Izadian and P. Famouri, "Fault diagnosis of MEMS lateral comb resonators using multiple-model adaptive estimators", IEEE Transactions on Control Systems Technology, vol. 18, no. 5, pp. 1233-1240, Sept. 2010.

[7] N. Deb and R. D. Blanton, "High-level fault modeling in surfacemicromachined MEMS" Design, Test, Integration, and Packaging of MEMS/MOEMS, Proceedings of SPIE, vol. 4019, pp. 228-235, May 2000.

[8] R. Reichenbach, R. Rosing, A. Richardson, and A. Dorey, "Finite element analysis to support component level fault modelling for MEMS," Design, Test, Integration, and Packaging of MEMS/MOEMS, Proceedings of SPIE, vol. 4408, pp. 147-158, April 2001.

[9] Z. Chen, Y. Y. He, F. L. Chu, and J. Huang, "Dynamic characteristic analysis of the micro-structure with defects," Chinese Journal of Mechanical Engineering, vol. 40, no. 6, pp. 23-27, 2004.

[10] S. Mir, B. Charlot, and B. Courtois, "Extending fault-based testing to microelectromechanical systems," Journal of Electronic Testing: Theory and Applications, vol. 16, no. 3, pp. 279-288, 2000.

[11] D. Paci, M. Mastrangeli, A. Nannini, F. Pieri, "Modeling and characterization of three kinds of MEMS resonators fabricated with a thick polysilicon technology", Analog Integr. Circ. Sig. Process., vol. 48, pp. 41-47, 2006.

[12] M. W. Putty, S. C. Chang, R. T. Howe, A. L. Robinson, K. D. Wise, "One-port active polysilicon resonant microstructures", Proc.IEEE Micro Electromechanical Systems, pp.60-65, Feb.1989.

[13] S. Chowdhury, M. Ahmadi, W. C. Miller, "Pull-in voltage calculations for MEMS sensors with cantilevered beams", IEEENEWCAS the 3rd International Conference, Québec City, Canada, vol. 19-22, pp.143 - 146, June 2005.

[14] K. Tanaka, R. Kihara, A. S. Amores, J. Montserrat, "Parasitic effect on silicon MEMS resonator model parameters", Proceedings of the IEICE General Conference, 2001.

[15] B. Chariot, S. Moussouris, S. Mir and B. Courtois, "Fault modeling of electrostatic comb-drives for MEMS", Symposium on Design. Test, and Microfabrication of MEMS and MOEMS, SPIE vol. 3680, Paris, France, 1999.

[16] S. A. Rittenhouse, "Diagnosis of Operational Changes in Microelectromechanical Systems via Fault Detection", Thesis submitted to the College of Engineering and Mineral Resources at West Virginya University, 2004. 


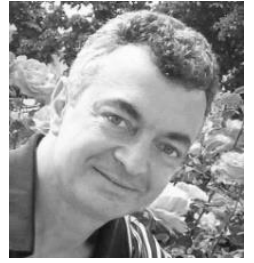

Serhat Ikizoğlu was born in Istanbul/Turkey in 1957. He received his BSc Degree from Istanbul Technical University (ITU), Electronics and Communication Engineering Department in 1980. He graduated from the same university, Control and Computer Engineering Department with MSc and $\mathrm{PhD}$ Degrees in 1982 and 1992 respectively. $\mathrm{He}$ is currently Assistant Professor in Control and Automation Engineering Department at ITU. His research interests are electrical measurement and instrumentation, mechatronics and biomedical engineering.

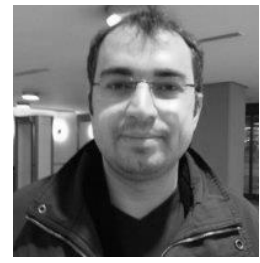

Muhammed Akyol received his BSc Degree from the Electronics and Telecommunication Engineering at Istanbul Technical University in 2004. He graduated from Electrical Engineering Department in Sept. 2008. He worked at Turkish Airlines Technique Inc. for 4 years.

He is currently a pilot at Turkish Airlines. 